\title{
Three-Dimensional Transient Electromagnetic Forward Modeling In Direct Time-Domain by Vector Finite Element
}

\author{
$\mathrm{He} \mathrm{Li,} \mathrm{Xiu} \mathrm{Li} \mathrm{*,}$ \\ College of Geology Engineering and \\ Geomatics ,Chang'an University, Xi'an 710054, China \\ Email :1030839536@qq.com
}

\author{
Weihua Yao \\ China Coal Technology \&Engineering Group XI'AN \\ Research Institute, Xi'an 710077, China
}

\begin{abstract}
In this paper, the direct time domain vector finite element method was used to forward three-dimensional transient electromagnetic fields excited by loop source. From Maxwell's equations met by time domain electromagnetic field we can directly export the double curl equations met by electric field, and then get the variation of electric field equations. Take backward difference to discrete the time item, after the element analysis for all units in the calculation area, and synthesize all units to get the stiffness matrix. The sparse matrix is stored by the CSR format. This work introduces the source current density into the double curl equations met by electric field. We use a trapezoidal waveform in the excitation source and taking the switch on-time, on-time and switch off-time into consideration. A loop source is added in modeling. In fact, this method makes the models arbitrarily complex in resistivity distribution. In the process of solving the equations, take the whole time zone segmentation process strategy, to take a unified iterative steps within the section to improve the computational efficiency. Then we use the direct time-domain vector finite element mothed to calculate and verify the uniform half-space model, shallow water undulating terrain model and the marine low resistance model.
\end{abstract}

Keywords-transient electromagnetic method; threedimensional forward modeling; direct time-domain by vector finite element mothed; ramp time

\section{INTRODUCTION}

In recent years, transient electromagnetic method received wide attention for its economy, non-destructive, fast, high precision, and information-rich and other unique advantages. Especially the three-dimensional transient electromagnetic forward modeling got the highly anticipated for its fine response law of complicated probe target. Currently, threedimensional transient electromagnetic forward modeling methods include finite difference method, finite volume method, integral equation method and finite element method. Compared to other types of methods, finite element method has the advantage of the applicability of the complex physical parameters distribution and irregular geometric distribution. With the rapid development of computer hardware, the computational problem has been effectively alleviated, therefore the finite element method is considered to be a promising forward mothed. Three-dimensional transient electromagnetic finite element method forward generally includes two categories. One is the direct method, to use differential format discrete time items. Then we solve the electromagnetic field in the time domain directly. The other is the indirect method, to obtain frequency-domain electromagnetic field, and then transformed it into the time domain. The accuracy of the second mothed depends on the time-frequency conversion method, and it is limited to the way of the source added in the modeling, and then we can't forward a variety of different transmit waveforms. Therefor we choose direct time-domain vector finite element mothed to forward the transient electromagnetic fields. And we can achieve three-dimensional transient electromagnetic full waveform forward.

\section{THEORY AND METHOD}

\section{A. Equations}

Under quasi-static, isotropic, non-dispersive, zoning uniform medium, ignore the displacement current, the timedomain Maxwell equation can be expressed as follows,

$$
\begin{aligned}
& \nabla \times \vec{E}=-\mu_{0} \frac{\partial \vec{H}}{\partial t} \\
& \nabla \times \vec{H}=\sigma \vec{E}+\vec{J}_{s} \\
& \nabla \cdot \vec{E}=0 \\
& \nabla \cdot \vec{H}=0
\end{aligned}
$$

Get the electric field equation as follows,

$$
\nabla \times \nabla \times \vec{E}+\sigma \mu_{0} \frac{\partial \vec{E}}{\partial t}+\mu_{0} \frac{\partial \vec{J}}{\partial t}=0
$$

In this paper, we use the first class Dirichlet boundary condition at the infinite boundary. In other words, electric field intensity's or magnetic field intensity's tangential component is zero at the infinite boundary.

We obtain the variational equations as follows,

$$
\int_{V} f \cdot\left(\nabla \times \nabla \times \vec{E}+\sigma \mu_{0} \frac{\partial \vec{E}}{\partial t}+\mu_{0} \frac{\partial \vec{J}}{\partial t}\right) d V=0
$$

Then, we simplify (3) as follows, 


$$
\begin{aligned}
& \int_{V}\left[(\nabla \times f) \cdot(\nabla \times \vec{E})+\sigma \mu_{0} f \cdot \frac{\partial \vec{E}}{\partial t}\right. \\
& \left.+\mu_{0} f \cdot \frac{\partial \vec{J}}{\partial t}\right] d V=0
\end{aligned}
$$

Discreting the time item as follows,

$$
\frac{\partial \vec{E}_{k}}{\partial t_{k}}=\frac{\vec{E}_{k}-\vec{E}_{k-1}}{t_{k}-t_{k-1}} \quad(k=2, \cdots, n)
$$

Substituting (5) to (4),

$$
\begin{aligned}
& \int_{V}\left(\nabla \times N_{i}\right) \cdot\left(\nabla \times N_{j}\right) d V \vec{E}_{k}+\sigma \mu_{0} \int_{V} N_{i} \frac{\vec{E}_{k}}{t_{k}-t_{k-1}} d V \\
& =\sigma \mu_{0} \int_{V} N_{i} \frac{\vec{E}_{k-1}}{t_{k}-t_{k-1}} d V-\mu_{0} \int_{V} N_{i} \frac{\vec{J}_{k}-\vec{J}_{k-1}}{t_{k}-t_{k-1}} d V
\end{aligned}
$$

Then we write (6) in the matrix form as follows,

$$
\begin{gathered}
\mathbf{A}_{\mathbf{e}} \mathbf{E}_{\mathbf{e}}^{\mathbf{k}}=\mathbf{b}_{\mathbf{e}} \\
\mathbf{A}_{\mathbf{e}}=\int_{V}\left[\left(\nabla \times N_{i}\right) \cdot\left(\nabla \times N_{j}\right)+\frac{\sigma \mu_{0}}{t_{k}-t_{k-1}} N_{i} \cdot N_{j}\right] d V \\
\mathbf{b}_{\mathbf{e}}=\frac{\sigma \mu_{0}}{t_{k}-t_{k-1}} \int_{V} \vec{E}_{k-1} \cdot N_{i} d V- \\
\frac{\mu_{0}}{t_{k}-t_{k-1}} \int_{V}\left(\vec{J}_{k}-\vec{J}_{k-1}\right) \cdot N_{i} d V
\end{gathered}
$$

After the element analysis for all units in the calculation area, then generate the stiffness matrix by the overall synthesis of the units. Then we can get large sparse linear equations. The sparse matrix is stored by the CSR format.

\section{B. Load Source}

This work introduces the source current density into the double curl equations met by electric field. We use a trapezoidal waveform in the excitation source and taking the switch on-time, on-time and switch off-time into consideration. Fig.1 shows a typical trapezoidal wave schematic diagram.

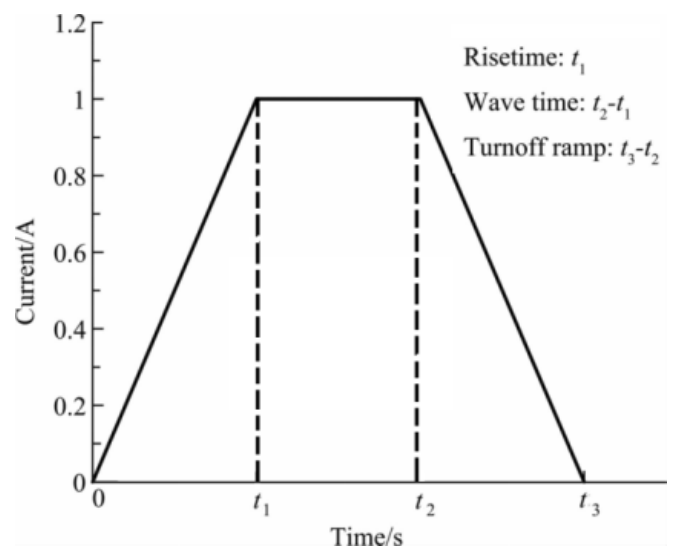

Fig.1. Trapezoidal wave schematic diagram

\section{Solving equations}

In this paper, the equations are solved by the parallel solver PARDISO in the MKL library, since the calculation process requires a lot of iterations, to shorten single-step calculation time and reduce the number of iterations are two key questions. The process of solving the equations, time is mainly spent in the stiffness matrix Cholesky decomposition. Equations left item only concerned with iterative step. If control the iterative step unchanged, the equations left item unchanged. It is not necessary to decomposed stiffness matrix each step, to use the results the previous step calculate. This method can greatly improve the efficiency of single-step calculation. At the same time, taking into account current dramatic changes in the switch on-time and switch off-time. In order to ensure the stability, iterative step requires very little, if the whole process adopted the iterative step, it will inevitably result in dramatic increase in the number of iteration. Therefore, considering the single-step calculation time and iteration number, take the whole time zone segmentation process strategy, to take the unified iterative steps within the section to improve the computational efficiency.

\section{Model CAlculation}

In order to verify the correctness of the method and the reliability of the program, we calculate the uniform half-space model, shallow water undulating terrain model and the marine low resistance model.

\section{A. The algorithm validation}

To verify the accuracy of the algorithm, we use the uniform half-space model to verify. The resistivity of the model is $100 \Omega \cdot m$. The size of the square loop source is $100 \mathrm{~m} \times 100 \mathrm{~m}$, the electricity is $1 \mathrm{~A}$, the switch on-time and the switch off-time are $1^{\mu s}$, and the on-time is $5 \mathrm{~ms}$. Fig. 2 shows the comparison between results of time-domain vector finite element method and those of digital filtering method of uniform half-space model and the relative error.

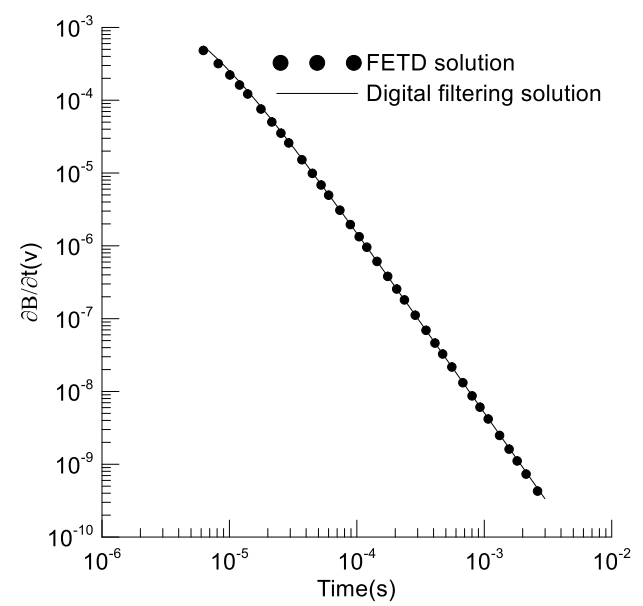

(a) 


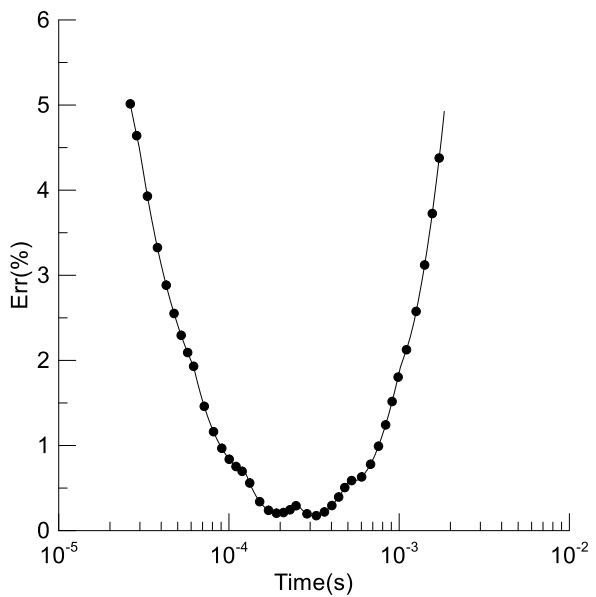

(b)

Fig.2. Test of direct time-domain vector finite element method with the uniform half-space model

(a)Comparison between results of the direct time-domain vector finite element method and those of digital filtering method of uniform half-space model (b) Relative error of forward modeling results of uniform half-space model

Fig.2 shows that the results between direct time-domain vector finite element method and the response of digital filtering method fit better. And the relative error keeps within $5 \%$, which shows the accuracy of the method in this paper.

\section{B. Shallow water undulating terrain model}

Coastal zone is the most concentrated area of human activity, most of the world's population and cities are concentrated in the coastal zone. Affected by human activities, shallow sea area terrain changes rapidly. It has important practical significance to determine the underwater bottom topography quickly on economic construction and scientific research. Then designed two underwater bottom topography model based on actual data in this paper, geoelectric parameters of the model are shown in Fig.3 and Fig.5, and the magnetic moment is $256000^{A-m^{2}}$.

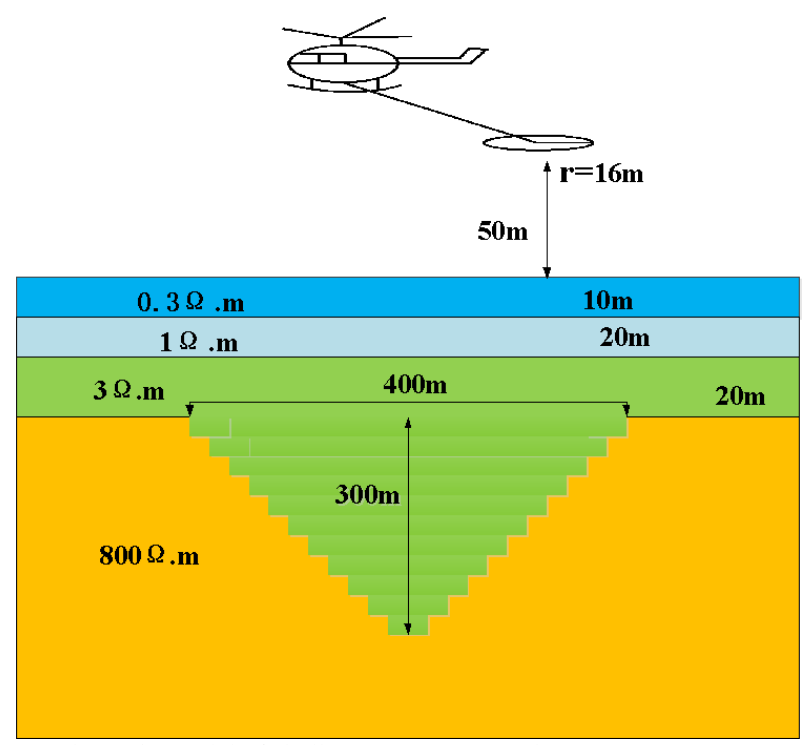

Fig.3. Schematic model of shallow water topography

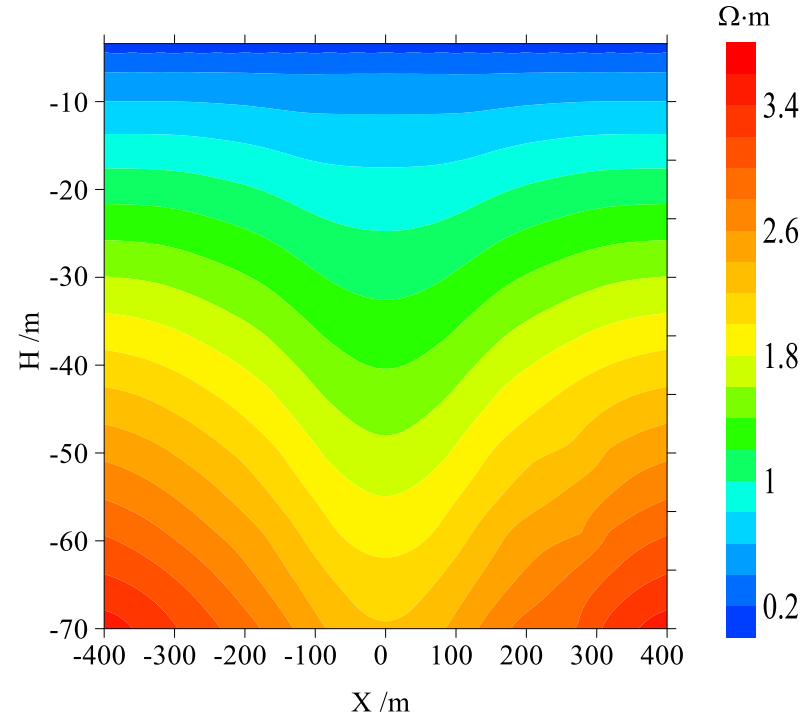

Fig.4. Apparent resistivity section diagram

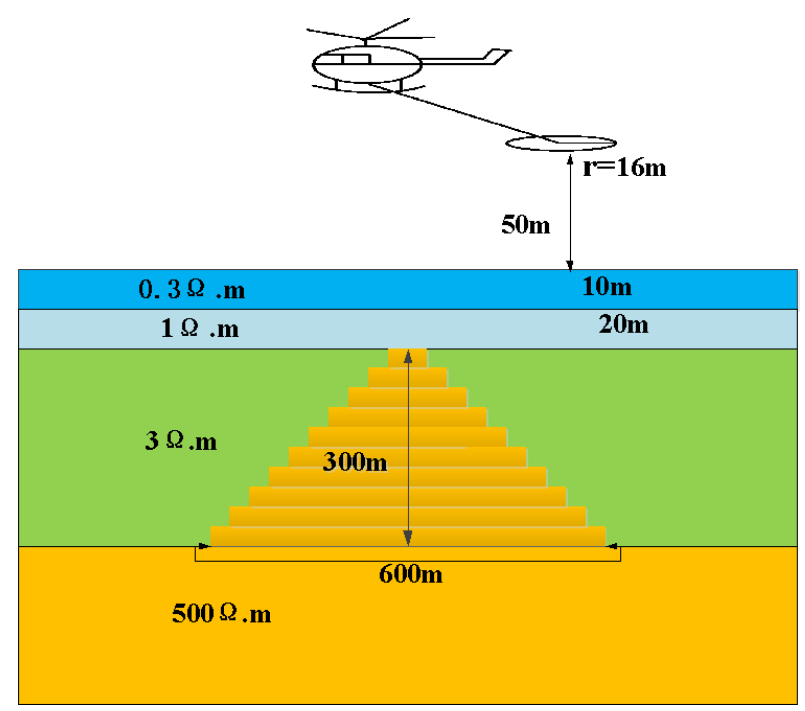

Fig.5. Schematic model of shallow water topography 


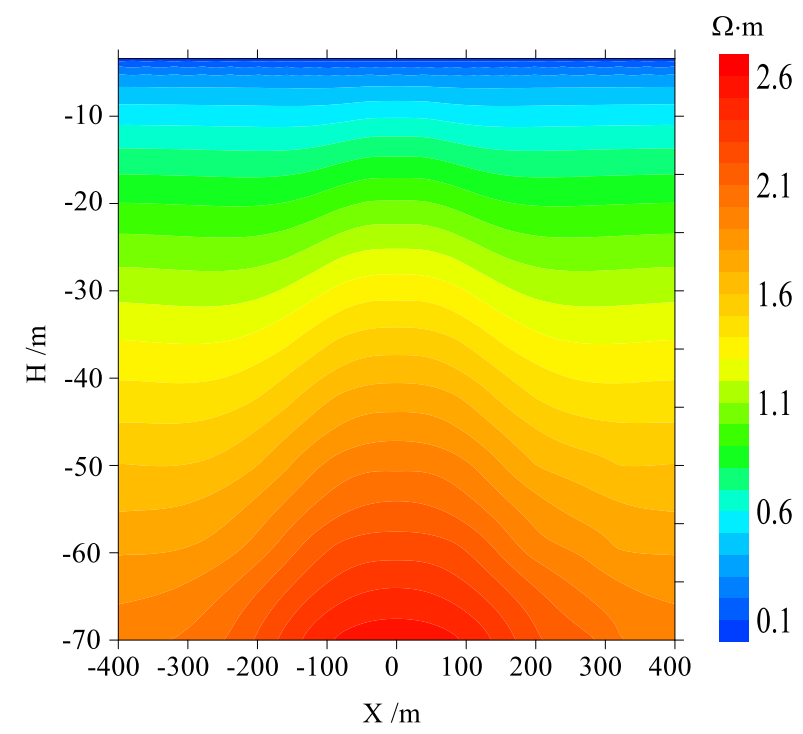

Fig.6. Apparent resistivity section diagram

Apparent resistivity profile (Fig.4, Fig.6) can clearly reflect the topography, and it is consistent with the design model, which illustrates the reliability of three-dimensional forward modeling for transient electromagnetic by direct timedomain vector finite element method. And it can provide accurate topographical data of the shallow coastal area for the economic development and the scientific research.

\section{The marine low resistance model}

The marine low resistance model calculations, geoelectric parameters of the model are shown in Fig.7, and the magnetic moment is 16 million $A-m^{2}$.

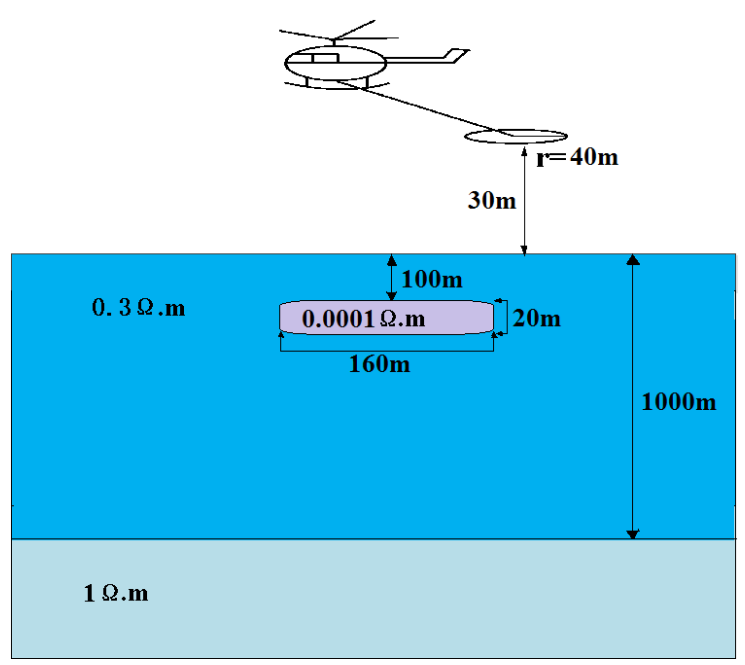

Fig.7. Schematic model of the marine low resistance model

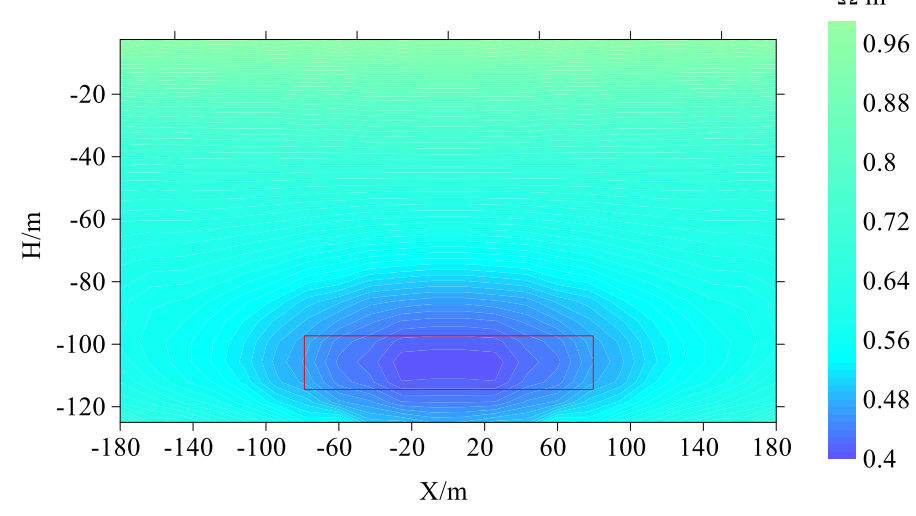

Fig.8. Apparent resistivity section diagram

The apparent resistivity section diagram is consistent with the schematic model. Further explained it is reliability that using the direct time-domain vector finite element method for simulating 3D transient electromagnetic field.

\section{CONCLUSION}

(1) To use the direct time-domain vector finite element method for simulating 3D transient electromagnetic field. And this method has broader applicability to add the source into the model.

(2) To use a trapezoidal waveform in the excitation source and taking the switch on-time, on-time and switch off-time into consideration, and we can achieve three-dimensional transient electromagnetic full waveform forward for arbitrarily complex model.

(3) To take the whole time zone segmentation process strategy, to take the unified iterative steps within the section to improve the computational efficiency.

\section{REFERENCES}

[1] LI Jian-hui, ZHU Zi-qiang, ZENG Si-hong, et al,2012, Progress of forward computation in transient electromagnetic method. Progress in Geophys. (in Chinese), 27(4),1393-1400. 